\begin{tabular}{|l|l|l|l|}
\hline $\begin{array}{l}\text { Eiszeitalter und Gegenwart } \\
\text { Quaternary Science Journal }\end{array}$ & $\mathbf{5 6 / 4}$ & 283-294 & Hannover 2007 \\
\hline
\end{tabular}

\title{
Zur Struktur und Entstehung von Eiskeil-Großformen in Lieth/Elmshorn (Schleswig-Holstein)
}

\author{
Alf Grube $^{*}$
}

\begin{abstract}
Kurzfassung: Die oberflächennahen Ablagerungen des Perm und Quartär im Westteil der Liether Kalkgrube sind intensiv periglaziär überprägt. Drei annähernd parallel verlaufende, bis zu mehr als $3 \mathrm{~m}$ tiefe und 0,5 $\mathrm{m}$ breite Eiskeil-Pseudomorphosen sind aufgrund der Ausbildung in den Zechstein-Aschen und -Kalken, der ungewöhnlichen Größe und der Vergesellschaftung mehrerer ähnlich großer Strukturen von besonderer Bedeutung. Ihre Entstehung lässt sich mit den spezifischen Standortfaktoren erklären. Eine feinkörnige Beschaffenheit des Wirtsgesteines bot gute Voraussetzungen für eine intensivere Frostwirkung, ebenso wie die Exposition der Zechstein-Aschen mit geringer Quartärbedeckung. Eine ungewöhnlich große mechanische Festigkeit der Zechstein-Aschen im Vergleich zu typischen norddeutschen Lockergesteinen begünstigte die Entstehung größerer Strukturen. Die Vergesellschaftung, die ähnliche Dimensionierung und ähnliche Orientierung der drei großen Eiskeile deuten auf einen Zusammenhang mit einer salinartektonischen Vorzeichnung hin. Die sedimentologisch-tektonische Struktur der halokinetisch überformten Zechstein-Ablagerungen bestimmte die äußere Geometrie der Eiskeile maßgeblich mit. Bei der Füllung hinsichtlich Struktur, Mächtigkeit der einzelnen Füllungsabschnitte, Bodenart und -farbe deuten sich zwei unterschiedliche Bildungsphasen an. Offenbar erfolgte bei zwei Strukturen eine randliche, einseitige Öffnung und Verfüllung. Die Strukturen stellen möglicherweise prä-weichselkaltzeitliche Bildungen dar.
\end{abstract}

[Structure and development of ice-wedge pseudomorphs in the Lieth lime quarry/Elmshorn (Schleswig-Holstein)]

\begin{abstract}
The deposits near the surface of the Permian and Quarternary in the western part of the "Lieth lime quarry" have been intensively periglacially modified. Three ice-wedge pseudomorphs, which run approximately parallel, are $0.5 \mathrm{~m}$ wide and reach a depth of more than 3 meters. These features are of particular importance to periglacial science due to their setting in Zechstein-ashes and -limestones, their unusual size and their spatial relationship to several similarly large structures. Their development can be explained by geological factors. The fine-grained calcarious rocks, within which the wedges are developed, offer good conditions for intensive frost action, in a similar fashion to the exposed Zechstein rocks with their shallow overburden of quaternary material. An unusually large mechanical strength of the surrounding ashes compared with sedimentary rocks elsewhere in northern Germany favoured the emergence of larger structures. Spatial relationships, similar dimensions and orientation of the three large ice wedge pseudomorphs point to a connection with salt-tectonic structures. The structure of the latter had a considerable impact on the overall geometry of the ice wedges. Field relationships between structure, thickness, soil type and colour of individual fill units suggests two different phases of formation: A one-sided opening and backfilling appears to have taken place within two structures. The likely age of the reported structures possibly pre-dates the Weichselian glaciation.
\end{abstract}

Keywords: ice-wedge, periglacial, karst formation, salt tectonics

\footnotetext{
*Anschrift des Verfassers: Dr. A. Grube, Landesamt für Natur und Umwelt des Landes Schleswig-Holstein, Abt. Geologie und Boden, Dezernat Geologie, Hamburger Chaussee 25, 24220 Flintbek. email: agrube@lanu.landsh.de
} 


\section{Einleitung}

Der Aufschluss Liether Kalkgrube befindet sich im Geestbereich auf dem Rundhorst Elmshorn, einem doppelsalinaren Salzdiapir im Südwesten Schleswig-Holsteins (Abb. 1). Die Oberfläche der Salzstockgesteine (Oberrotliegend und Zechstein) ist generell stark reliefiert, sie ist durch differentielle Salzstockhebung, Salinartektonik i. e. S., Verkarstung sowie durch glazigene und periglaziäre Prozesse geprägt worden (GRUBE 1997). Das meist geringmächtige Deckgebirge besteht überwiegend aus quartären Ablagerungen. Tertiäre Sedimente finden sich nur lokal als Füllungen in subrosiv gebildeten Hohlformen.

Im Mai 2005 wurden bei der Erstellung eines Schurfes im Westteil der Liether Kalkgrube quartäre Ablagerungen im Hangenden von Zechsteinablagerungen angetroffen. Die oberflächennahen permischen und quartären Ablagerungen sind intensiv periglaziär überprägt.

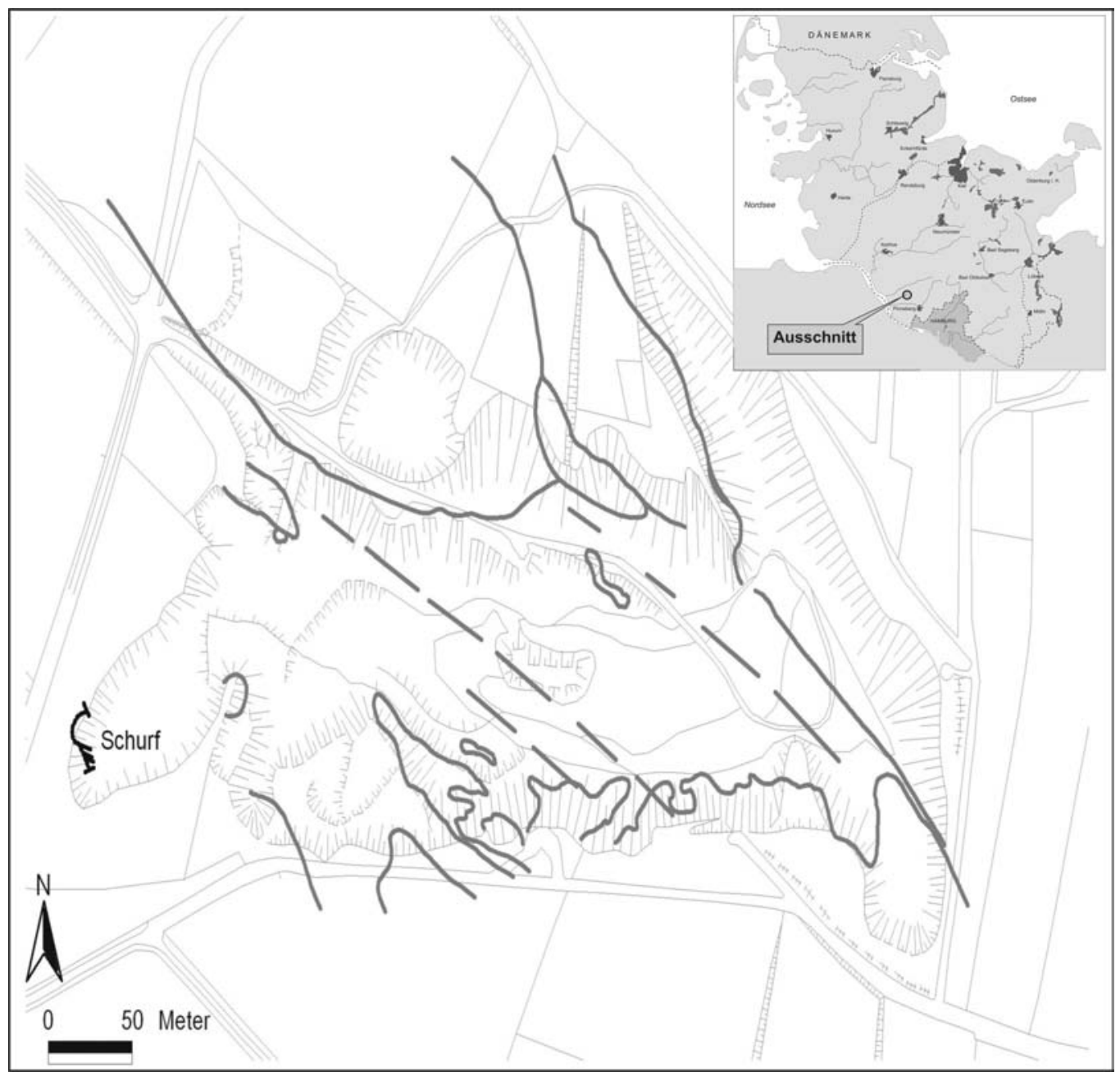

Abb. 1: Lageplan des Schurfes am Westrand der Liether Kalkgrube mit Verlauf der drei Eiskeile. Gestrichelte Linien zeigen Störungen nach F. Grube (1957), MenKe (1975) und VolLmer (1988).

Fig. 1: Location of the cut at the western end of the Lieth quarry with the three ice wedges. Dashed lines show faults after F. Grube (1957), MenKe (1975) und Vollmer (1988). 
Von besonderer Bedeutung sind drei annähernd parallel verlaufende, bis zu mehr als 3 Meter tiefe und $0,5 \mathrm{~m}$ breite Eiskeilpseudomorphosen, die die anstehenden Zechstein-Ablagerungen durchschlagen. Aufgrund ihrer Größe und ihrer spezifischen Struktur sind diese von überregionaler Bedeutung. Die im Schurf aufgeschlossenen Sedimente und Strukturen wurden hinsichtlich ihrer Verteilung, ihrer petrographischen Zusammensetzung, ihrer Genese und ihres Alters charakterisiert. Hierbei lag ein Schwerpunkt auf den ungewöhnlichen EiskeilPseudomorphosen. Im Folgenden werden die Begriffe Eiskeil-Pseudomorphose und Eiskeil synonym verwendet. Entsprechend ihrer Lage wird bei den drei aufgeschlossenen Eiskeilen von südlichem, mittlerem und nördlichem Eiskeil gesprochen.

\section{2 Übersicht der aufgeschlossenen Schichtenfolge im Schurf}

Die untersuchte Schichtenfolge liegt im Bereich einer lokalen Perm-Aufragung. Diese taucht zu den Seiten unter Ablagerungen der Elster- und der Saale-Kaltzeit (Till der älteren Saale-Kaltzeit) ab, die wiederum flächenhaft von Flugsanden überlagert werden. Die Geländehöhe im Bereich des Schurfes liegt zwischen ca. $+10,5$ und $+12 \mathrm{~m} \mathrm{NN}$. Die Kuppenposition mit verringerten Quartär-Mächtigkeiten von Dezimetern bis zu wenigen Metern konnte durch Sondierbohrungen flächenhaft nachgewiesen werden. Bei den angetroffenen Zechstein-Ablagerungen dominieren Kalk-Aschen. Diese können als sekundäre chemische Ausfällung der kalkreichen Schichtglieder des Zechsteins angesehen werden (MENKE et al. 1984). Die Kalk-Aschen wurden in den vergangenen Jahrzehnten für die Herstellung von Düngekalken verwendet. Zum Liegenden hin nimmt der Anteil von Kalksteinen und Stinkschiefern, die teilweise brecciös auftreten, zu.

Die Zechstein-Aschen sind vorwiegend schluffig zusammengesetzt, lokal treten auch tonige Bereiche auf $(n=7$; Mittelwerte: $65 \%$ Schluff, $24 \%$ Sand, $7 \%$ Ton und $3 \%$ Kies). Bei Vernachlässigung zweier verhältnismä-
Big sandiger Proben steigt der Mittelwert des Schluffanteils auf ca. $75 \%$. Mit ebenfalls $75 \%$ dominierte der Feinsandanteil deutlich an der Sand-Gesamtfraktion. Die Zechstein-Aschen sind grau bis dunkelgrau gefärbt. Insgesamt sind vielfach Zechsteinkalke und Stinkschiefer mit fließenden Übergängen in die ZechsteinAschen eingeschaltet. Lokal finden sich auch Einsprenglinge von Gips bzw. Marienglas. Calcit $\left(\mathrm{CaCO}_{3}\right)$ und - untergeordnet - Dolomit $\left(\mathrm{CaMg}\left(\mathrm{CO}_{3}\right)_{2}\right)$ sind die deutlich vorherrschenden Mineralphasen in den Zechstein-Aschen (freundliche schriftl. Mitt. Ludwig, Min.-Petrog. Inst. Univ. Hamburg).

Bestimmungen des Porenanteils (n) aus 11 ungestörten Proben erbrachten Werte zwischen 37 und 46,3\% mit einem Mittelwert von 40,2 \%. Der Wassergehalt (w) lag im Durchschnitt bei $18,4 \%$. Der Mittelwert der Korndichte ( $\rho)$ aus 14 Proben lag bei $2,73 \mathrm{~g} / \mathrm{cm}^{3}$. Die aus einaxialen Versuchen ermittelten Druckfestigkeiten (qu) liegen für 4 Proben im Schurf bei einem Mittelwert von $115 \mathrm{kN} / \mathrm{m}^{2}$. Flügelsondierungen im Aufschluss ergaben um ca. 10 bis $20 \%$ höhere horizontale als vertikale Messergebnisse. Zechsteinkalke und Stinkschiefer treten massiv südlich und nördlich der Eiskeile auf, wo sie teilweise die Oberfläche der Perm-Ablagerungen bilden (vgl. Abb. 2). Die intensive salinartektonisch bedingte Verstellung, die alle Zechsteinablagerungen betrifft, wird in den kompetenten Zechstein-Kalken deutlich. VoLLMER (1988) ermittelte im Westen der Liether Kalkgrube eine generelle NW-SE-Orientierung von Schichtflächen, Längsklüften, Störungen, Falten und Schuppen. Die Einmessung der im Schurf sichtbaren Verwerfungen passt in dieses Bild.

Die quartären Ablagerungen im Schurf erreichen (außerhalb der drei großen Eiskeile) Mächtigkeiten von bis zu ca. 3,5 m. Die maximale Mächtigkeit wird in einer kleinräumigen Hohlform erreicht, deren Flanken steil und deren Basis uneben ist. Diese Hohlform ist mit einem Till-ähnlichen Sediment verfüllt, welches vorwiegend Perm-Gesteine enthält. Örtlich tritt ein braunrot gefärbter Lokal-Till auf, der äußerlich dem Esing-Till (vgl. VINX et al. 1997) 


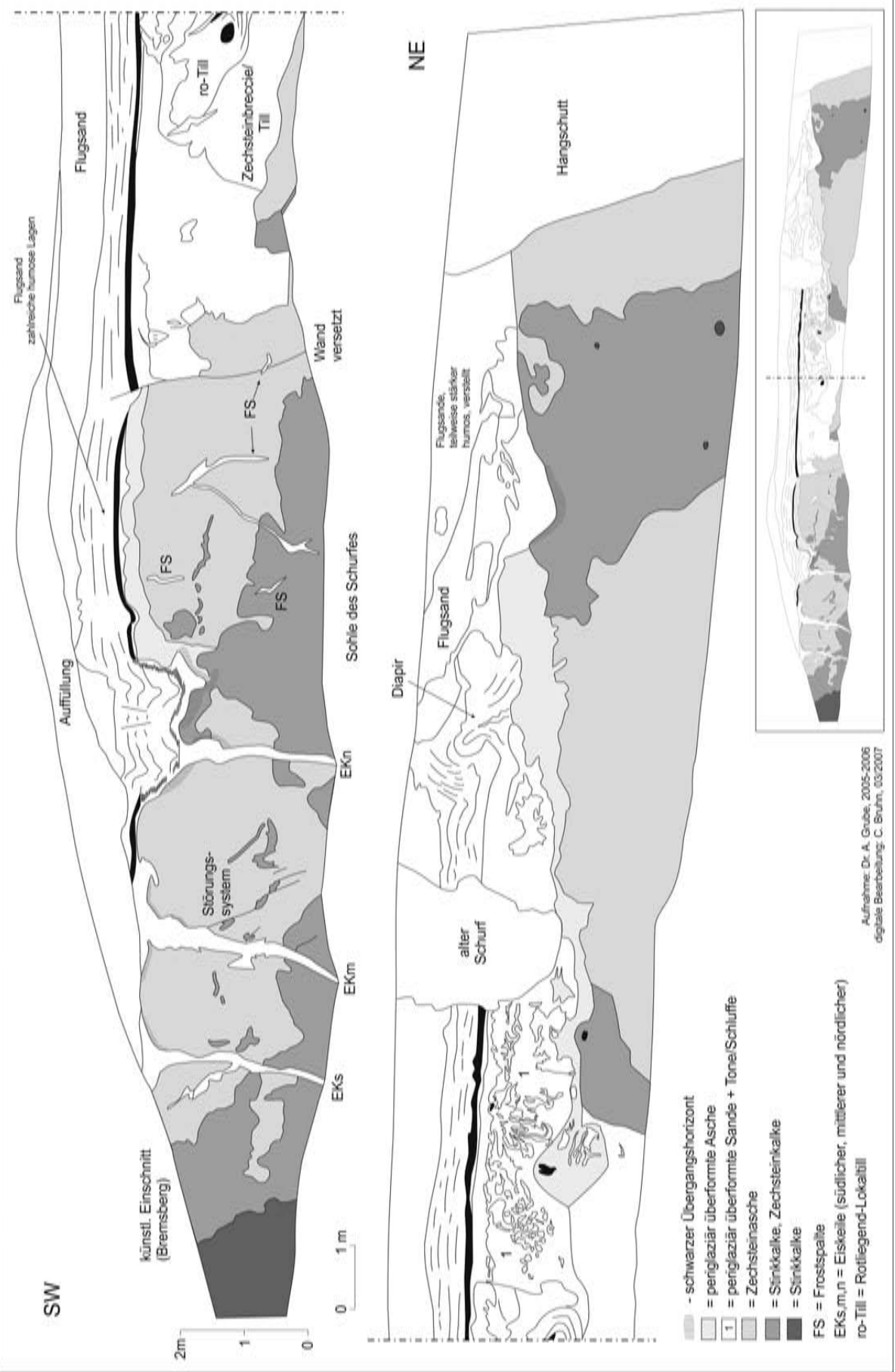

Abb. 2: Übersichtsabbildung der im Schurf aufgeschlossenen Strukturen und Ablagerungen.

Fig. 2: General view of structures and sediments exposed in the cut. 
gleicht. Der Till enthält viel Zechstein- sowie Rotliegend-Material. Er ist erwartungsgemäß ausgesprochen kalkreich. Teilweise treten in der Hohlform Wechsellagerungen zwischen hellen Sanden und dunkelgrau gefärbten Schluffen auf, letztere sowie die oberen Bereiche des Till-ähnlichen Sedimentes sind intensiv periglaziär verbrodelt. Eine periglaziäre Überprägung ist auch durch zahlreiche steilgestellte Geschiebe gut zu erkennen. Dunkle Horizonte in den „Verwürgungen“ bzw. Brodelböden sind vorwiegend aus Zechsteinmaterial aufgebaut. Geschiebe bis zu 0,35 m sind an der Oberkante der Kryoturbations-Schicht bzw. an der Unterseite der hangenden „Sand-Pakete“ zu finden. Im Hangenden des gesamten Schurfes (einschließlich des Bereiches mit Eiskeilen) treten ca. 1,0 m mächtige, in der unteren Hälfte deutlich geschichtete, fein- und mittelsandige Flugsande auf. Diese sind bei unmittelbarer Auflagerung auf den kryoturbaten Ablagerungen teilweise in die periglaziäre Überprägung einbezogen, z. B. durch zapfenförmige Einsinkungen. Im nordöstlichen Bereich des Schurfes sind die Verstellungen besonders stark. Die Basis der geschichteten Fein- und Mittelsande bildet oft ein ca. 0,1 m mächtiger dunkelbrauner, stärker humoser Horizont. Dieser kann nach pollenanalytischen Bestimmungen von Dr. habil. H. Usinger (Kiel) in das Meiendorf gestellt werden. Nach Usinger (2007) fehlen Wärme liebende Gehölze in diesem Horizont vollständig, ein Sanddornwert von annähernd $25 \%$ (bezogen auf die Landpflanzenpollensumme) weist auf das Meiendorf-Interstadial hin. Er entspricht dem „Hippophaë-Horizont“ von Menke (in Bokelmann et al. 1983) in einer benachbarten archäologischen Grabung. Im Hangenden wechseln hellgraue Sande mit geringmächtigen dunkelbraunen, stärker humosen Lagen ab. Den Abschluss bilden weitgehend ungeschichtete, beige-farbene Fein- und Mittelsande.

Im nordöstlichen Bereich des Schurfes ist ein größerer Diapir bzw. eine gekippte Falte vorhanden, die die hangenden geschichteten Flugsande verstellt hat. Der Diapir enthält vorwiegend schluffig-sandiges Material, es kommen auch kleine Schuppen von Ton vor. Er ist auf der oberen Seite durch zwei grabenartige Einbrüche zerteilt. Der Diapir wird hier als periglaziäre Bildung angesehen (vgl. HAMMEN \& MAARlEVEld 1952; DüCKer 1954). Östlich des Diapirs setzt eine Hohlform ein, die durch eine wechselhafte Folge von Sanden und humosen Abschnitten gekennzeichnet ist und vermutlich durch Abbauaktivitäten überformt ist.

Im hangenden Abschnitt des nördlichen Eiskeils ist ein Bereich von ca. 1,2 x 2 m grabenförmig eingebrochen (Abb. 3). Diese Form geht in einen westlich angrenzenden, vermutlich im Rahmen des Kalkascheabbaus angelegten, ehemaligen Schurf über, der im Frühjahr 2007 sichtbar wurde. Die breitere Ausbildung des Mündungshalses des Eiskeils ist offenbar subrosiv überprägt. Der Eiskeil ist im Bereich einer bis kurz unter die Erdoberfläche aufragenden Kalksteinaufragung ausgebildet.

Die Zechsteinablagerungen im Schurf sind vielfach durch Frostspalten zerschlagen, die meist nur wenige Zentimeter breit sind. Diese

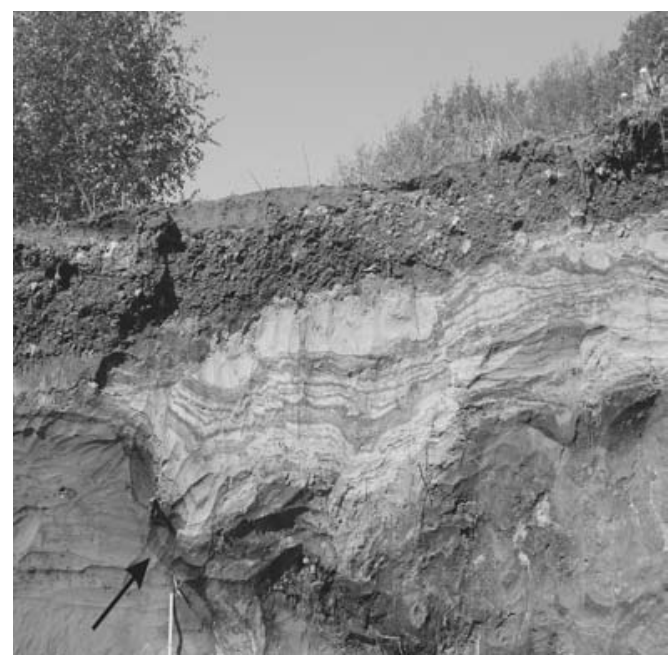

Abb. 3: Top des nördlichen Eiskeils (Zollstockende $=0,2 \mathrm{~m}$ als Maßstab); vermutlich durch Subrosion verstellte Flugsande. Am Übergang zu den Zechstein-Aschen tritt ein mächtiger schwarzer Horizont auf (Pfeil).

Fig. 3: Top of the northern ice wedge (rule $=0,2 \mathrm{~m}$ as scale); dislocated drift sands. At the transition to the Zechstein-ashes a thick black layer occurs (arrow). 
Frostspalten reichen - wenn sie unabhängig von den drei großen Eiskeilen auftreten - bis zu ca. 1,5 m unter die Erdoberfläche. Teilweise deutet sich dabei eine Orientierung an vorhandenen Störungen an. Die Füllung besteht meist aus hellgrauen bis gelblichen Fein- und Mittelsanden, die erwartungsgemäß kalkreich sind. Im Bereich der massiven schwarzen Kalksteine bzw. Stinkschiefer, unmittelbar benachbart zum südlichen Eiskeil, zeigen sich unregelmäßig eingelagerte quartäre, gelbliche Sande. Diese dürften periglaziär eingetragen worden sein, vermutlich durch eine Art Eislinsen-Wachstum. Auffälligste Erscheinungen der periglaziären Überprägung sind drei große Eiskeile.

\section{Feldbefunde}

\subsection{Position, Form und Füllung der Eiskeile}

Die drei großen Eiskeile liegen angenähert parallel zueinander. Der südliche und der mittlere Eiskeil verlaufen, bei einer leichten Neigung von 5 bis $10^{\circ}$ nach Süden, annähernd gleichsinnig, der nördliche dagegen ist senkrecht orientiert. Der mittlere Eiskeil ist der größte. Die Breite erreicht maximal ca. 0,5 Meter (vgl. Abb. 4 und 6). Die Eiskeile sind noch bis zu mehr als 0,2 $\mathrm{m}$ breit an der Basis des aufgeschlossenen Bereiches, d. h. sie dürften sich noch mehrere Meter unter die derzeitige Grubensohle hin fortsetzen. Alle drei Strukturen sind nicht typisch langgestreckt-keilförmig geformt wie viele Eiskeile in Norddeutschland, sondern, im Verhältnis zu ihrer Tiefe, verhältnismäßig schmal und unregelmäßig in ihrer Gestalt. Der mittlere Eiskeil zeigt im Fußbereich eine Aufteilung in einen Haupt- und einen Nebenstrang (Abb. 4). Vielfach treten hakenförmige Absätze auf. Die seitlichen, eckig gestalteten Flanken der Eiskeile finden sich teilweise in direkter Fortsetzung vorhandener Störungen in den Zechstein-Ablagerungen.

Die Orientierung der drei Eiskeile in der Fläche konnte anhand der östlichen Fortsetzung der Eiskeile nach Ost-Südost an der Sohle des Schurfes (vgl. Abb. 1 und 5) sowie an- hand einer kleineren Aufgrabung einige Meter westlich der aufgeschlossenen Wand mit etwas mehr als ca. $100^{\circ}$ festgestellt werden. Dieses passt zu der von VolLmer (1988) und älteren Bearbeitern ermittelten generellen NW-SEOrientierung von Schichtflächen, Längsklüften, Störungen, Falten und Schuppen in der Liether Kalkgrube.

Die Eiskeile sind vorwiegend durch eine sandig-feinkörnige Füllung gekennzeichnet, wobei der Anteil schluffigen Materials nach unten hin zunimmt. Der mittlere Eiskeil ist generell durch einen höheren Sandanteil charakterisiert. Die sandigen Bereiche zeigen im Durchschnitt $86 \%$ Sand, $11 \%$ Ton und Schluff sowie $2 \%$ Kies. Teilweise ist in Abschnitten der Eisspalten ein „,coarsening upwards“, von Schluff zu Mittelsand, zu erkennen, z. B. im südlichen und mittleren Eiskeil (Mitte). Die Breite der einzelnen breiteren Verfüllungseinheiten variiert von wenigen Zentimetern bis $\mathrm{zu}$ ca. $15 \mathrm{~cm}$, ihre Länge erreicht häufig mehrere Dezimeter. Im oberen Teil des linken und mittleren Eiskeils treten bevorzugt hellgraue Sande auf; sonst sind beigefarbene, braune und dunkelbraune Sande dominierend. Die die Eiskeile ausfüllenden Sedimente sind grundsätzlich weit gestuft. Dabei sind die Füllungen meist verhältnismäßig lang, häufig erreichen sie 0,5 m und mehr. Im nördlichen Eiskeil findet sich ein zentrales dunkles, feinkörniges Band, welches fast über den gesamten aufgeschlossenen vertikalen Anschnitt verläuft.

Im unteren Teil sind alle drei Eiskeile zu einem größeren Anteil mit feinsandigem bis schluffigem Material gefüllt. Im südlichen und mittleren Eiskeil treten diese bindigeren Ablagerungen an den Flanken, in allen drei Eiskeilen im unteren aufgeschlossenen Bereich flächenhaft auf. Die bindigen Ablagerungen sind nur in wenigen Bereichen leicht Humus-führend. Die Verteilung und Größe der Kalkgehalte sind unregelmäßig. Der Kalkgehalt liegt bei durchschnittlich 1,4 \%. Die quartären Sande sind meist kalkfrei. Die bindigen quartären Füllungsbereiche sind dagegen i. d. R. stark kalkhaltig, maßgeblich durch aufgearbeitetes Zechsteinmaterial. Teile des insgesamt durch 


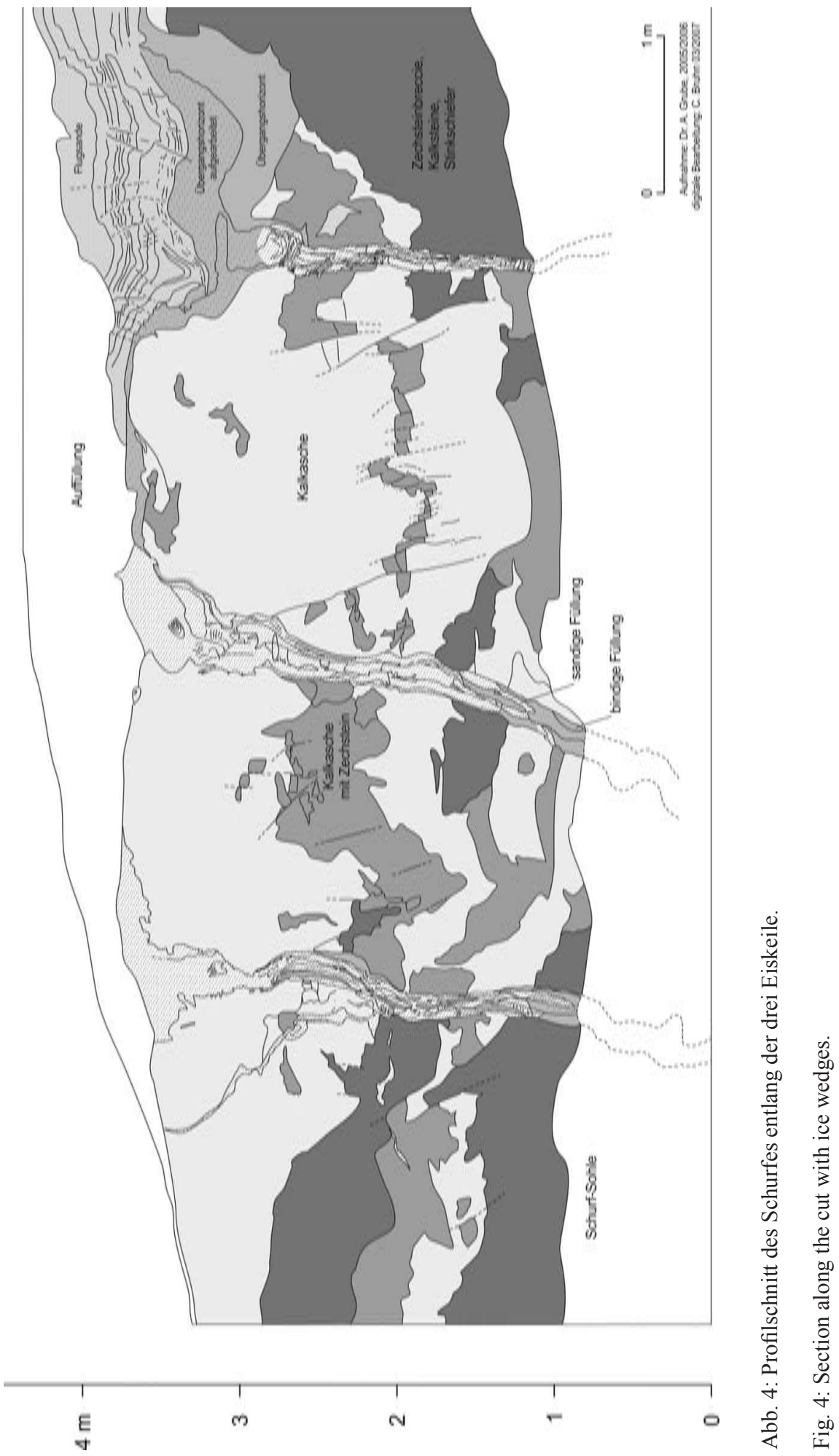




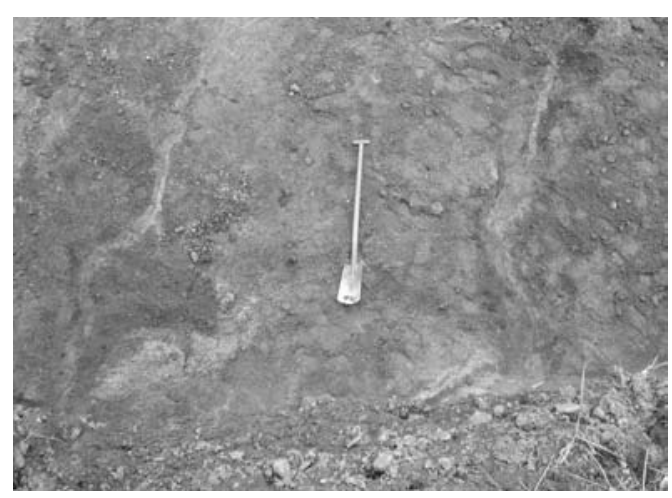

Abb. 5: Aufsicht auf die Fortsetzung des südlichen (rechts) und mittleren Eiskeils in die Fläche nach Ost-Südost (liegender Spaten als Maßstab). Erkennbar sind die unregelmäßige Breite der Strukturen sowie deren seitliche Verästelungen.

Fig. 5: Areal view on the southern (right) and middle ice wedge to the east-southeast (spade for scale). Visible are the irregular width of the structures and the lateral treeing.

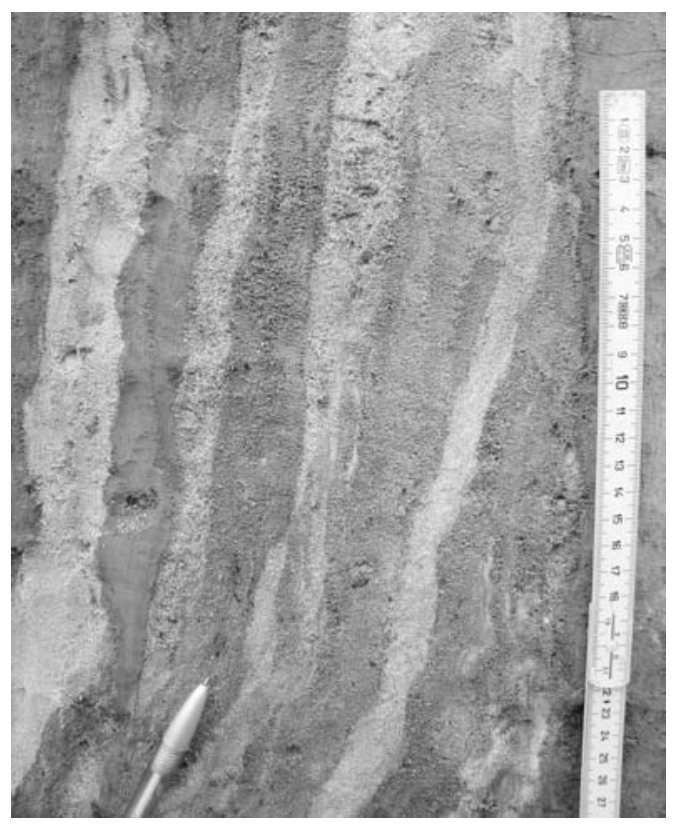

Abb. 6: Parallel-schichtiger Aufbau des mittleren Teils des südlichen Eiskeils. Eine vertikalsymmetrische Füllung fehlt.

Fig. 6: Parallel layering of the middle part of the southern ice wedge. A vertical symmetric filling is missing. einen höheren Sandanteil charakterisierten mittleren Eiskeils sind generell kalkärmer. Lokal treten in den Eiskeilen Zechsteinschlieren, Zechsteingerölle und -schollen auf. Diese können teilweise Durchmesser bzw. Längen von einigen Dezimetern erreichen (vgl. Abb. 6). Die Füllungen sind verhältnismäßig arm an Makrofossilien. Es treten jedoch z. B. Schneckenreste (im mittleren Eiskeil) von Turitella tricarinata (Obermiozän, Oberes Langenfeldium / Gramium; Bestimmung Dr. K. Gürs 03.01.2006) auf. Diese stammen vermutlich aus den Pinneberger Schichten, die ehemals flächenhaft im Bereich verbreitet gewesen sein dürften.

Charakteristische rotbraune bis braune, lokal auch schwarze Verfärbungen treten durch Eisen- und Mangan-Ausfällungen in allen drei Eiskeilen auf. Sie kommen bevorzugt in den Randbereichen zur benachbarten Zechsteinasche, den Stauwasserbereichen (bei Abknicken der Strukturen und Unterlagerung durch Zechsteinasche) und in den unteren, feinkörnigeren Abschnitten der Eiskeile vor. Auch die benachbarten schmalen Eisspalten zeigen vielfach Eisen-Ausfällungen.

\subsection{Kontaktbereich der Eiskeile zum umgebenden Gestein}

Die Oberfläche der Zechstein-Aschen einschließlich der benachbarten Zechsteinablagerungen (Zechsteinkalke usw.) bildet im Bereich der Eiskeile sowie im nördlich benachbarten Abschnitt des Schurfes eine unregelmäßige Aufragung. Dabei ist die Mächtigkeit der quartären Auflagerung über der Zechsteinasche gering. Auffällig ist eine schwarze und kalkfreie, tonige Reaktionszone am Übergangsbereich zwischen den chemisch sauer reagierenden Sanden und den alkalischen Zechstein-Aschen. Muskovit und Mikroklin (untergeordnet Quarz) sind die vorherrschenden Mineralphasen in diesem Horizont (Mitt. Hr. Ludwig), der seine größte Mächtigkeit mit ca. 0,3 m im Bereich des subrosiv überformten nördlichen Eiskeils erreicht. In den anderen beiden Eiskeilen ist er nur ca. 1-2 cm mächtig. Ein entsprechendes Band tritt in geringer Mächtigkeit auch 
im nördlichsten Bereich des Schurfes auf, wo eine flache Einsenkung in der Zechsteinasche vorhanden ist. Der Horizont fehlt an den Überhängen, kommt also nur bei einem stärkeren Durchtritt von Sickerwasser aus den Sanden in die Zechsteinasche bzw. bei einer entsprechenden Stauwasserbildung auf den Zechstein-Aschen vor. In einem Streifen von bis zu wenigen Dezimetern von den Eiskeilfüllungen entfernt treten auch in den Zechstein-Aschen Farbveränderungen auf. Die Kalk-Aschen sind in diesen Bereichen etwas aufgehellt (grau statt dunkelgrau), teilweise durch Eisenoxide auch rötlichbraun verfärbt.

\subsection{Periglaziäre Überprägung der Eiskeile}

Die Eiskeile zeigen, vorwiegend im obersten verbreiterten Abschnitt, eine deutliche periglaziäre Überprägung. Es finden sich Verlaufsstrukturen ähnlich Brodelböden, taschenartige Strukturen sowie diapirartige Verstellungen der angrenzenden Zechsteinasche. Im mittleren und rechten Eiskeil sind vorwiegend die oberen ca. 0,8 $\mathrm{m}$ im breitesten angeschnittenen Abschnitt kryoturbat überprägt. Unterhalb treten nur in Teilbereichen kryoturbationsartige Verstellungen auf. Erwartungsgemäß sind auch in den den Eiskeilen benachbarten Zechstein-Aschen Kryoturbations-Erscheinungen zu beobachten.

\section{Interpretation}

Die hier diskutierten Strukturen werden aufgrund der Füllung mit heterogenen quartären Ablagerungen als Eiskeile gedeutet und nicht als salinartektonisch bedingte Bildungen, ältere Trockenrisse o. ä. Hierfür sprechen u. a. die Füllung und die intensive Verzahnung der Strukturen mit benachbarten Frostspalten- und -rissen. Eiskeile bilden sich bei niedrigen Temperaturen, wenn sich Kontraktionsrisse im Sediment bilden. Das Ausmaß dieser Rissbildung hängt vom Wirtsgestein, von der Exposition und der Einwirkungsdauer des Frostes ab. Der Prozess läuft zeitweise mehrphasig ab, in dem beim Auftauen Sedimentmaterial eingetragen wird. Dieser Transport kann fluviatil, durch
Rutschung oder äolisch erfolgen. Das Tauwasser kann erneut frieren und zu einer Erweiterung der Spalte führen.

Die Eiskeile in Lieth sind nicht im Sinne von Blume et al. (1979) unter kaltem Klima im direkten Randbereich des Inlandeises (schneefrei und extrem trocken bedingt durch Fallwinde des Eises) als reine Kontraktionsrisse gebildet worden, die anschließend ausschließlich mit Flugsand verfüllt wurden. Ihre Füllungen sowohl hinsichtlich der Körnung, d. h. bindige Anteile besonders im unteren Teil sowie auftretende größere Zechsteingerölle, als auch der Sedimentstruktur (teilweise einseitiges Aufreißen) deuten vielmehr auf eine Eiskeilbildung hin.

Bei der Bildung der beschriebenen Eiskeile haben die besonderen Standortfaktoren eine Rolle gespielt. Das Alter der Eiskeilbildungen bleibt unklar. Wenngleich eine Bildung während der Weichsel-Kaltzeit aufgrund der geringmächtigen Überlagerung mit Flugsanden nahe liegt, ist auch eine möglicherweise erheblich frühere Bildung denkbar. Für eine ältere Bildung sprechen u. a. die bindigen Sedimente im unteren Bereich der Eiskeile und die Anteile von Tertiär-Material. Anhand des Fossilgehaltes ist eine Aufarbeitung von miozänem Material nachweisbar. Diese Aufarbeitung weist ggf. auf ein höheres als weichsel-kaltzeitliches Alter der Eiskeile hin, da heute tertiäre Ablagerungen in der Umgebung fehlen. Das geologische Umfeld mit vorherrschenden Ablagerungen der Elsterund der Saale-Kaltzeit stützt diese Auffassung. Der schwarze Reaktionshorizont könnte ein weiterer Hinweis auf ein höheres Alter sein, da entsprechende mineralogische Verwitterungsreaktionen längere Zeit beanspruchen.

Die geringe Mächtigkeit quartärer Ablagerungen über der Zechsteinasche hat vermutlich bei der Entstehung der massiven Eiskeile eine große Rolle gespielt. Die während der Bildung der Eiskeile weitgehend freiliegende Aufragung der Zechstein-Aschen ermöglichte eine ungestörte Frosteinwirkung, die die Ausbildung großer Eiskeile förderte. Die ungewöhnliche Größe der Eiskeile dürfte maßgeblich mit den spezifischen bodenmechanischen Eigenschaften des durchschlagenen Zechsteinmaterials in 
Zusammenhang stehen. Die Zechstein-Aschen sind verhältnismäßig gleichmäßig zu ca. $90 \%$ aus Ton, Schluff und Feinsand zusammengesetzt. Die notwendigen Temperaturen zur Bildung von Eiskeilen sind in feinkörnigem oder organischem Material generell geringer anzusetzen als in rolligem Material. So wird von Romanovsky (1985) ein mindestens $\mathrm{zu}$ erreichender Temperaturwert von $-2^{\circ} \mathrm{C}$ für Schluffe angegeben, im Vergleich zu $-5,5^{\circ} \mathrm{C}$ bei Sanden und Kiesen (vgl. DIN 18196). Die Kalk-Aschen des Zechsteins mit ihrem weit dominierenden Anteil der Schluff-Fraktion boten somit gute Voraussetzungen für eine längere Bildung von Eiskeilen.

Die ermittelten einaxialen Druckfestigkeitswerte in den Zechstein-Aschen sind als verhältnismäßig hoch anzusehen. Die hohe Festigkeit dürfte die mechanische Zerstörung der Zechsteinablagerungen erheblich begünstigt haben. Die gemessenen horizontalen Druckfestigkeiten (Flügelsonde) liegen um ca. 10 bis $20 \%$ über den vertikalen, wenngleich eine primäre Schichtung in den Zechstein-Aschen makroskopisch nur lokal zu erkennen ist. Die Kalke des Zechsteins stehen bezüglich ihrer Druckfestigkeitswerte am Übergang zum Festgestein und wurden daher hier nicht näher untersucht.

Eine wichtige Rolle dürften das relativ große Porenvolumen und der erhöhte Wassergehalt der Zechstein-Aschen spielen. Nach herrschender Lehrmeinung findet die Eiskeilbildung vorwiegend in ,nassen“ Regionen wie Deltas, Senken usw. statt (WASHBURN 1979). Die feinkörnige Ausbildung des Wirtsgesteins mit entsprechendem Wasserhaltevermögen in Verbindung mit der Lage der Eiskeile im Bereich der morphologischen Aufragung der Zechstein-Aschen, die einen bevorzugten Eingriff von Frost ermöglichten, begünstigten die Bildung großer Eis-Strukturen.

Der Grund für die Unregelmäßigkeit des vertikalen Gesamtverlaufs der Eiskeile sowie ihre hakenförmigen Versätze sind überwiegend auf die salinartektonisch bedingte Struktur des durchschlagenen Zechstein-Materials (Asche und Zechsteinkalke) zurückzuführen. Absätze bzw. Versätze folgen in ihrer Orientierung häufig auch Schichtungsfugen, die deutliche Inhomogenitäten darstellen (vgl. Abb. 7). Ein größerer fluviatiler Einfluss ist dagegen bei der Formung der Eiskeilumrisse nicht zu erkennen. An den Flanken der Eiskeile treten hakenförmige Absätze auf, an deren Enden sich kleinere Frostspalten in das benachbarte Zechsteinmaterial fortsetzen.

Die Vergesellschaftung, die ähnliche Dimensionierung sowie die ähnliche Orientierung der drei großen Eiskeile zeigen einen Zusammenhang der lateralen äußeren Gestalt der Eiskeile mit salinartektonischen Vorzeichnungen (besonders südlicher und nördlicher Eiskeil). Beide Strukturen sind im Randbereich der Breccien-Aufragungen „durchgeschlagen“. Bei dem mittleren Eiskeil ist kein Zusammenhang mit kompetenten Zechsteinablagerungen $\mathrm{zu}$ erkennen. Die Fortsetzung der Eiskeile in

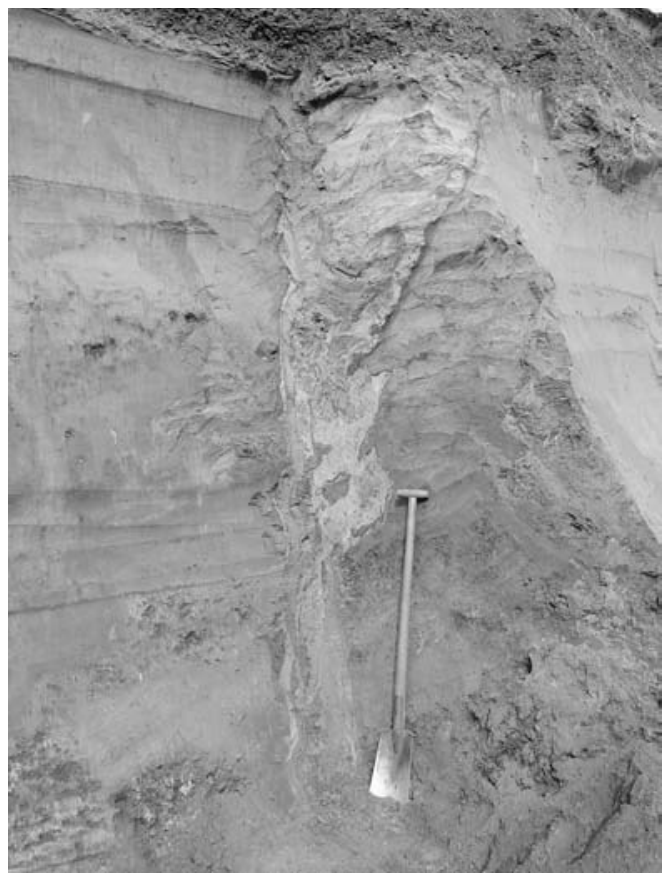

Abb. 7: Mittlerer Eiskeil während der Abgrabung; zentral Schollen von Zechsteinasche in pleistozäner Eiskeilfüllung; links unten im Bild Einschaltungen von Zechsteinkalken in der Kalkasche.

Fig. 7: Middle ice wedge during excavation; rafts of Zechstein ashes in pleistocene filling; Zechstein limestones incorporated in the ashes to the left. 
der Fläche mit verhältnismäßig geradliniger Orientierung ist ebenfalls ein Hinweis auf die Bindung an vorhandene Strukturen.

Das Sediment der Eiskeilfüllungen ist quartären Alters. Die Zusammensetzung des Ausgangsmaterials ist sehr variabel. In der Anfangsphase der Verfüllung der aufgeschlossenen Eiskeilbereiche wurde verstärkt bindigeres Material eingetragen. Der untere Teil der Eiskeile ist nicht nur generell feinkörniger, sondern auch insgesamt heterogener zusammengesetzt. Hier tritt zudem vermehrt horizontale Schichtung auf; ggf. sind somit zwei wesentliche unterschiedliche Bildungsphasen angedeutet.

Alle Strukturen zeigen - in den unteren, nicht durch Kryoturbation beeinflussten Bereichen - eine „mehrphasige“ Verfüllung durch parallele, mehr oder weniger senkrecht stehende Sedimentlagen an (vgl. Abb. 6). Die Spaltenfüllungen sind beim südlichen und mittleren Eiskeil überwiegend nicht parallel-symmetrisch gestaltet, vielmehr sind einseitig-singuläre Füllungseinheiten ausgebildet. Folglich kann davon ausgegangen werden, dass kein zentrales Aufreißen dieser Spalten erfolgt ist. Vielmehr erfolgte offenbar überwiegend eine randliche, einseitige Öffnung und Verfüllung. Beim nördlichen Eiskeil ist - zumindest im oberen Teil - ein zentrales Aufreißen dieses vertikal orientierten bzw. nicht geneigten Eiskeils zu erkennen. Die eher einheitliche Verfüllung einer Spalte in Bezug auf das Sediment sowie die verhältnismäßig große Längserstreckung der einzelnen Füllungseinheiten von oft mehr als $0,5 \mathrm{~m}$ spricht für eine schnelle Verfüllung der ehemaligen Spalten. Horizontalgeschichtete Füllungen treten in den Hintergrund, was ebenfalls für eine schnelle Verfüllung spricht.

Es treten in unterschiedlichem Maße fein verteilte Beimengungen von Zechstein-Ablagerungen auf. Die auftretenden Zechsteinschlieren, Zechsteingerölle und -schollen in den Eiskeilen sind in unterschiedlicher Weise zu interpretieren. Schlieren und Zechsteingerölle sind durch Aufarbeitung von Zechsteinablagerungen in der Umgebung der Eiskeile eingetragen worden. Größere Zechsteingerölle und -schollen dürften sowohl bei Öffnung der Eisspalten als auch bei deren Wiederverfüllung von den Wänden bzw. Überhängen abgerissen sein.

\section{Danksagungen}

Herrn Hartmut Usinger (Kiel) sei für die palynologische Datierung, Herrn Roland Vinx und Herrn J. Ludwig vom Mineralogisch-Petrographischen Institut der Universität Hamburg für die röntgenanalytischen Bestimmungen gedankt. Herrn Hans-Joachim Wohlenberg von der Kulturgemeinschaft Tornesch, Frau Kaczirek und Herrn Neumann (beide L-A. f. Straßenbau), Karl Gürs, Helmut Lange und Heinz Nebendahl (alle LANU) danke ich für die Durchführung bzw. Unterstützung bei Labor- und Feldarbeiten. Herr Rolf Hübner (Wedel) förderte die Arbeiten durch eine hochwertige Photo-Dokumentation. Herrn Christian Schlüchter (Bern) danke ich für konstruktive Anmerkungen zum Manuskript. Sven Lukas (London) sei für die Korrektur des Abstracts gedankt.

\section{Literatur}

Blume, H.-P., Hoffmann, R. \& Pachur, H.-J. (1979): Periglaziäre Steinring- und Frostkeilbildungen norddeutscher Parabraunerden. - Z. Geomorph. N.F., Suppl.-Bd. 33: 257-265; Stuttgart.

Bokelmann, K., Heinrich, D. \& Menke, B. (1984): Fundplätze des Spätglazials am Hainholz-Esinger Moor, Kreis Pinneberg. - Offa, Ber. Mitt. Urgeschichte, Frühgeschichte, Mittelalterarchäologie, 40: 199-239; Kiel.

DüCKER, A. (1954): Die Periglazial-Erscheinungen im holsteinischen Pleistozän. - Göttinger Geogr. Abh., 16: 5-54 + Anhang; Göttingen.

Grube, A. (1997): Geologie des Deckgebirges der Struktur Elmshorn (Schleswig-Holstein). - In: Berichte-Reports des Geologisch-Paläontologischen Institutes, Univ. Kiel, 87: 1-169; Kiel.

Grube, F. (1957): Das Oberflächenbild der Salzstöcke Elmshorn, Lägerdorf (Holstein) und Stade (Niedersachsen). - Mitt. Geol. Staatsinst., 26: 5-22; Hamburg.

Hammen, T., van der \& MaArleveld, G. C. (1952): Genesis and dating of the periglacial deposits at the eastern fringe of the Veluwe. - Geol. Mijnbouw, 14: 47-54; Utrecht.

MenKe, B. (1975): Vegetationsgeschichte und Florenstratigraphie Nordwestdeutschlands im Pliozän 
und Frühquartär - Mit einem Beitrag zur Biostratigraphie des Weichselfrühglazials. - Geol. Jb., A26: 3-151; Hannover.

Menke, B., Christensen, S., Grube, F. \& Ross, P.-H. (1984): Der Salzstock Lieth/Elmshorn und das Quartär von Westholstein, Teil I.- In: Degens, E. T., Hillmer, G. \&. Spaeth, C. (Hrsg.): Exkursionsführer Erdgeschichte des Nordsee- und Ostseeraumes: 445-465, Hamburg (Selbstverlag d. Geol.-Pal. Inst.).

RomanovskiJ, N.H. (1985): Distribution of recently active ice and soil wedges in the USSR. - In: Church, M. \& Slaymayer, S. (Hrsg.) (1985): Field and Theory: Lectures in geocryology: 154-165; Vancouver (University of British Columbia).
Usinger, H. (2007): Pollenanalytische Datierung der Proben aus der Kalkgrube Lieth. - 1 S. + Pollenzählung [unveröff.].

VinX, R., Grube, A. \& Grube, F. (1997): Vergleichende Lithologie, Geschiebeführung und Geochemie eines Prä-Elster-I-Tills von Lieth bei Elmshorn.- Leipziger Geowissenschaften, 5: 83-103; Leipzig.

Vollmer, T. (1988): Geologische Neuaufnahme der Liether Kalkgrube. - DFG-Abschlußbericht Th 126/15-2, 49 S. und Anhang; Hamburg [unveröff.].

WASHBURn, A. L. (1979): Geocryology.- A survey of periglacial processes and environments. -406 S., London (Arnold). 